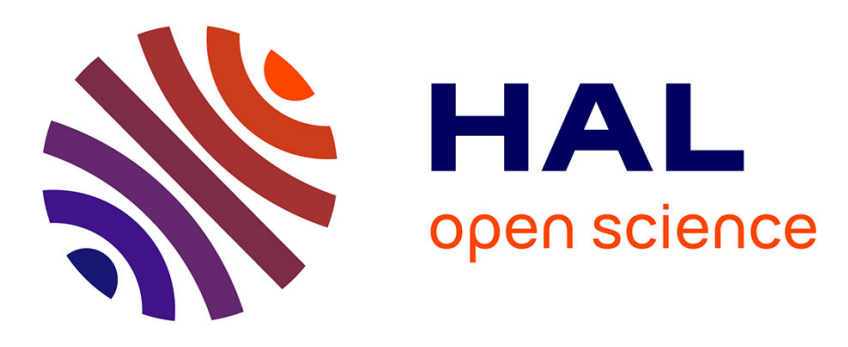

\title{
From in Situ HT-ESEM Observations to Simulation: How Does Polycrystallinity Affects the Sintering of CeO2 Microspheres?
}

\author{
G.I. Nkou Bouala, Nicolas Clavier, Sylvain Martin, Jacques Léchelle, Julien \\ Favrichon, H.P. Brau, Nicolas Dacheux, Renaud Podor
}

\section{To cite this version:}

G.I. Nkou Bouala, Nicolas Clavier, Sylvain Martin, Jacques Léchelle, Julien Favrichon, et al.. From in Situ HT-ESEM Observations to Simulation: How Does Polycrystallinity Affects the Sintering of CeO2 Microspheres?. Journal of Physical Chemistry C, 2016, 120 (1), pp.386-395. 10.1021/acs.jpcc.5b10465 . hal-01252614

\section{HAL Id: hal-01252614 \\ https://hal.science/hal-01252614}

Submitted on 2 Aug 2016

HAL is a multi-disciplinary open access archive for the deposit and dissemination of scientific research documents, whether they are published or not. The documents may come from teaching and research institutions in France or abroad, or from public or private research centers.
L'archive ouverte pluridisciplinaire HAL, est destinée au dépôt et à la diffusion de documents scientifiques de niveau recherche, publiés ou non, émanant des établissements d'enseignement et de recherche français ou étrangers, des laboratoires publics ou privés. 


\title{
From in situ HT-ESEM Observations to Simulation: How Does
}

\section{Polycristallinity Affects the Sintering of $\mathrm{CeO}_{2}$ Microspheres?}

\author{
G.I. Nkou Bouala ${ }^{1}$, N. Clavier ${ }^{1, *}$, S. Martin ${ }^{2}$, J. Léchelle ${ }^{3,4}$, J. Favrichon ${ }^{5}$, \\ H. P. Brau ${ }^{1}$, N. Dacheux ${ }^{1}$ and R. Podor ${ }^{1}$.
}

1. ICSM, UMR 5257 CEA/CNRS/ENSCM/Université de Montpellier, Site de Marcoule - Bât. 426, BP 17171, 30207 Bagnols/Cèze cedex, France

2. Ecole Nationale Supérieure des Mines, SPIN-EMSE, CNRS:UMR5307, LGF, 42023 Saint-Etienne,

France

3. CEA,DEN,DTEC,SECA,LCC, Site de Cadarache - Bât. 717, 13108 St-Paul lez Durance, France

4. CEA,DEN,DEC,SESC,LLCC, Site de Cadarache - Bât. 352, 13108 St-Paul lez Durance, France

5. CEA,DEN,MAR,UG-UST,STIC,GPSI, Site de Marcoule - Bât. 180, 30207 Bagnols/Cèze cedex, France

\section{* Corresponding author :}

Dr. Nicolas CLAVIER

ICSM - UMR 5257 CEA/CNRS/UM/ENSCM

Site de Marcoule - Bât 426

BP 17171

30207 Bagnols sur Cèze

France

Phone : + 33466339208

Fax : + 33466797611

nicolas.clavier@icsm.fr 


\section{Abstract}

The in situ observation of the first stage of sintering of cerium dioxide microspheres was performed using an environmental scanning electron microscope at high temperature (HTESEM). The associated morphological modifications were described quantitatively for systems constituted by two single crystal grains, on the one hand, and by two polycrystalline particles, on the other hand. Particularly, the in situ HT-ESEM observations, and subsequent image analysis with homemade image process software, led to assess the evolution of several parameters of interest during isothermal heat treatments, such as neck size, particles radii, dihedral angles between the spheres and distance between the grains centers. It was then possible to evaluate the activation energies associated to the neck formation for both systems studied, then to identify the different mechanisms involved. The diffusion process operating during the first stage of sintering was also pointed out. Furthermore, the comparison of the results obtained from polycrystalline particles and single crystals, and their confrontation with data coming from numerical computation, led to assess the influence of polycristallinity on the sintering kinetics. For all the conditions tested, sintering degree was found to be enhanced for polycrystalline particles, mainly because of the contribution of the mechanical rearrangement of crystallites during the neck's elaboration and of the existence of diffusion paths within the particles. On this basis, polycristallinity should be considered during numerical computations in order to provide predictive models for the first step of sintering.

\section{Keywords}

Sintering - In situ ESEM - Modelling - Cerium oxide - Polycrystalline - Single crystal 


\section{Introduction}

Sintering can be defined as the transformation of a powdered compact into a cohesive material under heating at high temperature ${ }^{\mathbf{1 , 2 , 3}}$. It appears as a key-step in the preparation of numerous technical ceramic materials as it drives both density and microstructure of the final objects, which could influence significantly the physico-chemical properties during their lifecycle. Sintering is usually described through three consecutive steps leading to the densification of ceramic materials. The initial stage involves the elaboration of necks between the grains and leads to the mechanical consolidation of the material ${ }^{4}$. The intermediate stage is then dedicated to the elimination of open porosity between the grains while the final one aims to the elimination of closed porosity through grain growth mechanisms.

The last two stages of sintering are mostly studied experimentally. Particularly, dilatometry and SEM observations are frequently used to monitor, quantify and understand the modifications undergone by the samples during their densification ${ }^{\mathbf{3 , 5}}$. Conversely, only few experimental works ${ }^{\mathbf{6}, 7}$ were until now devoted to the kinetics of neck elaboration because of the difficulties linked to the direct observation of a sample on a microscopic scale during a heat treatment. This stage is then generally mainly described through numerical simulations using simplified systems constituted by two spherical single crystals in contact ${ }^{\mathbf{8 , 9 , 1 0}}$. Among the various methods used to report the modifications of the sample during this stage of sintering, one can cite molecular dynamics ${ }^{\mathbf{1 1}}$; the Monte Carlo method ${ }^{\mathbf{1 2}}$ or the Discrete Elements Method $(\mathrm{DEM})^{\mathbf{1 3}}$. All of them involved some approximations on the grain morphology (supposed to be spherical) as well as on their crystalline state (single crystal) for the description of sintering. The geometrical assumptions can be avoided using a Monte Carlo method ${ }^{\mathbf{1 4}}$. However, the Monte Carlo time step which is used in these simulations is not related to physical time. Moreover, this method is not able to represent the competition between the different diffusion paths (surface, grain boundary and gas transport). Thus, the 
results obtained can hardly provide a robust and predictive description for the evolution of ceramic materials during the first stage of sintering. In these conditions, an experimental study of neck formation including direct observation of sample modifications should allow to point out the differences between modelling and experimental results, which could be linked to the hypothesis made in models, particularly concerning the polycristallinity of the samples.

In the present study, we report the first experimental observations of the initial stage of sintering for $\mathrm{CeO}_{2}$ microspheres by using Environmental Scanning Electron Microscopy at high temperature (HT-ESEM). The use of HT-ESEM allowed the in situ observation of the samples during long term heat treatments up to $1225^{\circ} \mathrm{C}$ under various atmospheres ${ }^{\mathbf{1 5 , 1 6 , 1 7}}$. Moreover, among other ceramic materials of interest, cerium oxide was targeted because of its wide range of applications, starting from its use in heterogeneous catalysis ${ }^{\mathbf{1 8}}$, but also as an additive for glasses, a stabilizer for $\mathrm{ZrO}_{2}$, a glass polisher ${ }^{19}$, or a surrogate material for $\mathrm{PuO}_{2}$ based nuclear fuels in the nuclear industry ${ }^{20}$.

More specifically, the study of cerium dioxide sintering was performed using both polycrystalline and single crystal particles, in order to compare the behavior of systems close to those used in models with real materials. In situ HT-ESEM studies were thus performed between $1000^{\circ} \mathrm{C}$ and $1225^{\circ} \mathrm{C}$ under air atmosphere. Processing the micrographs series allowed the quantification of several parameters of interest (grains and neck diameters, distance between the grains ...). Their variation versus temperature was then used to determine the mechanisms controlling the sintering of both systems and the corresponding activation energies. Finally, experimental data obtained for both kinds of particles studied were compared to results coming from mechanistic numerical simulations (using the SALAMMBO code), which led to evaluate the influence of polycristallinity on the sintering of $\mathrm{CeO}_{2}$ microspheres. 


\section{Experimental}

\subsection{Synthesis of polycrystalline and single crystal $\mathrm{CeO}_{2}$ microspheres}

The preparation of cerium oxide powders with spherical grains was performed through a wetchemistry route derived from the protocol reported in the literature by Minamidate et al. ${ }^{\mathbf{2 1}}$. During the synthesis, a $1.03 \mathrm{M}$ cerium (III) solution obtained by dissolution of $\mathrm{Ce}\left(\mathrm{NO}_{3}\right)_{3} .6$ $\mathrm{H}_{2} \mathrm{O}$ salt (supplied by Sigma-Aldrich) in deionized water was used as a starting reagent. 0.4 mole of cerium was then mixed with 0.025 mole of urea dissolved in water, used as a complexing reagent, leading to a final volume of about $50 \mathrm{~mL}$.

The as obtained mixture was first pre-aged at room temperature for 72 hours, then transferred into a Teflon-lined container and finally heated at $90^{\circ} \mathrm{C}$ for about 4.5 hours on a sand bath. The white precipitate obtained was collected by centrifugation at $4500 \mathrm{rpm}$, washed twice with deionized water and ethanol, and dried overnight in an oven at $60^{\circ} \mathrm{C}$. As already reported in our previous work, XRD pattern of the prepared powder advocated for the formation of cerium oxocarbonate $\mathrm{Ce}_{2} \mathrm{O}\left(\mathrm{CO}_{3}\right)_{2} \cdot n \mathrm{H}_{2} \mathrm{O}{ }^{22}$. Moreover, SEM observations revealed that the precipitate was made up of microspheres, with diameter ranging from 300 to $800 \mathrm{~nm}$ (Figure 1a). Polycrystalline $\mathrm{CeO}_{2}$ spheres could then easily be obtained from the conversion of these precursors above $400^{\circ} \mathrm{C}$ (Figure 1b).
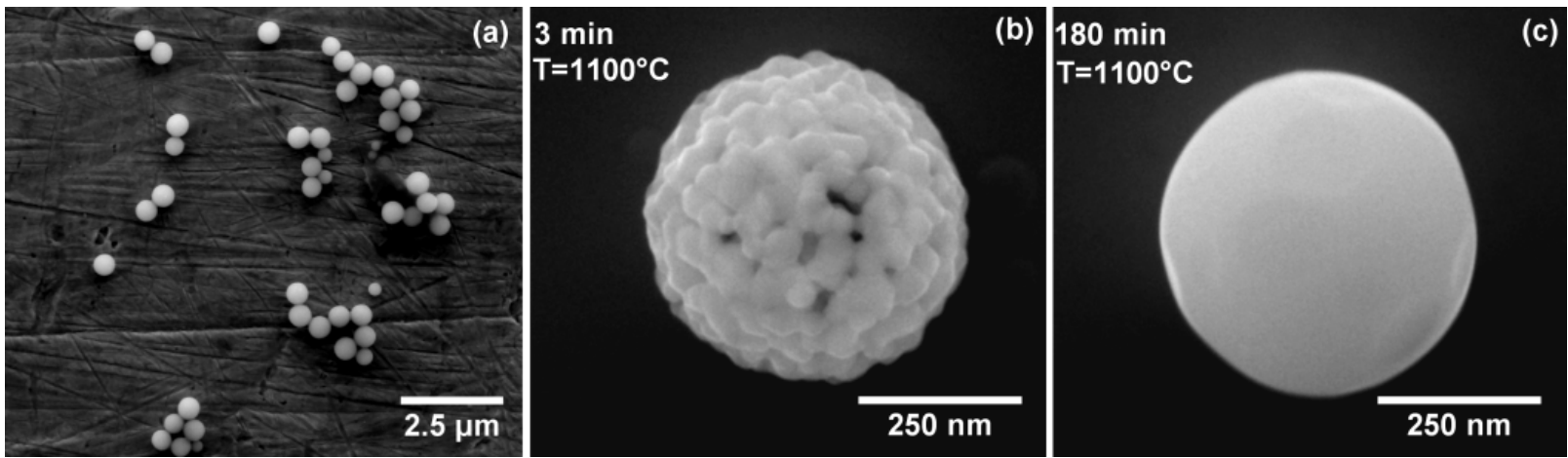

Figure 1 : SEM observations of the $\mathrm{CeO}_{2}$ microspheres dispersed on a Pt-Au10 thin foil (a). Details of the polycrystalline particles obtained after $3 \mathrm{~min}$ of isothermal heat treatment at $1100{ }^{\circ} \mathrm{C}(\mathrm{b})$ and of single crystal microspheres prepared after $180 \mathrm{~min}$ of isothermal heat treatment at $1100{ }^{\circ} \mathrm{C}(\mathrm{c})$. 
Moreover, heating of cerium oxide microspheres at high temperatures (typically above $1000^{\circ} \mathrm{C}$ ), led the number of crystallites constituting the microspheres to decrease gradually 22,23. As the initial morphology was systematically retained (Figure 1c), it was then possible to prepare spherical $\mathrm{CeO}_{2}$ single-crystals thanks to a heat treatment at $1100^{\circ} \mathrm{C}$ during 3 hours.

\subsection{Sample preparation}

Prior to their observation during in situ HT-ESEM experiments, all the samples were prepared according to the following procedure. First, $5 \mathrm{mg}$ of cerium oxide microspheres were dispersed in $1 \mathrm{~mL}$ of acetone; a drop of this mixture was then deposited at the surface of a 2 $\mathrm{mm}^{2} \mathrm{Pt}-\mathrm{Au} 10$ thin foil. This substrate was specifically selected in order to remain chemically inert on the whole range of temperature investigated (i.e. $1000^{\circ} \mathrm{C}-1225^{\circ} \mathrm{C}$ ) in order not to influence significantly the microstructural evolution of the sample during the sintering experiments.

The SEM observations of the surface of the Pt-Au10 thin foil where the $\mathrm{CeO}_{2}$ microspheres have been deposited show a good dispersion of the grains forming spontaneously systems constituted by two or more grains in contact (Figure 1a). The systems composed of two grains were used for the experimental investigation of first stage of sintering with polycrystalline and single crystal grains.

\subsection{HT-ESEM experiments}

The in situ high temperature experiments were performed with a FEI Quanta 200 fieldemission ESEM (Environmental Scanning Electron Microscopy) equipped with a $1500{ }^{\circ} \mathrm{C}$ hot stage ${ }^{\mathbf{1 6 , 2 4}}$. For the sintering of $\mathrm{CeO}_{2}$ microspheres, the HT-ESEM experiments were realized under air atmosphere at an operating pressure ranging between $200 \mathrm{~Pa}$ and $250 \mathrm{~Pa}$. After the 
sample preparation, the Pt-Au10 thin foil was transferred into the furnace directly attached in the microscope chamber. The sample was then heated at about $40^{\circ} \mathrm{C} \cdot \mathrm{min}^{-1}$ up to a temperature ranging between $1000^{\circ} \mathrm{C}$ and $1225^{\circ} \mathrm{C}$, then maintained on an isotherm all along the experiment. In the case of the sintering of polycrystalline grains, a $15 \mathrm{~min}$. holding time was carried out at $700^{\circ} \mathrm{C}$ in order to convert the precursors into oxide. When the targeted temperature was reached, secondary electron mode images were recorded continuously with a frequency of 2 to 60 images per minute during all the heat treatment using a gaseous secondary electron detector (GSED) ${ }^{\mathbf{2 5}}$.

\subsection{Image processing}

The SEM images recorded in situ were then processed for the determination of characteristic data. Image processing was performed following a two-step process. First, the extraction of the grain contours was processed using the Fiji software ${ }^{\mathbf{2 6 , 2 7}}$ and the specific Trainable Weka Segmentation plugin ${ }^{28}$. Then, these images were used for the direct determination of several parameters generally computed in the two grains models describing the morphological modifications occurring during the first stage of sintering. To achieve this goal, homemade software ImageJu was developed ${ }^{\mathbf{2 9}}$ and allowed the determination of such parameters from the segmented images. With this method, neck and grain radii, distance between the centers of the grains, neck radius of curvature and dihedral angles were directly determined (Figure 2).

The associated error attached to these values was found to depend on the heating time. Indeed, at the start of the isothermal plateau, the image appeared to be unstable because of external perturbations due to temperature stabilization. At this step, the images were then recorded with a scanning rate of $1 \mathrm{~s}$ and the relative error on the data obtained through images analysis was evaluated between $15 \%$ and $20 \%$. This error further decreased gradually during the 
experiment with the stabilization of the image, and reached about $5 \%$ for scanning rates comprised between 5 and $10 \mu \mathrm{s}$.

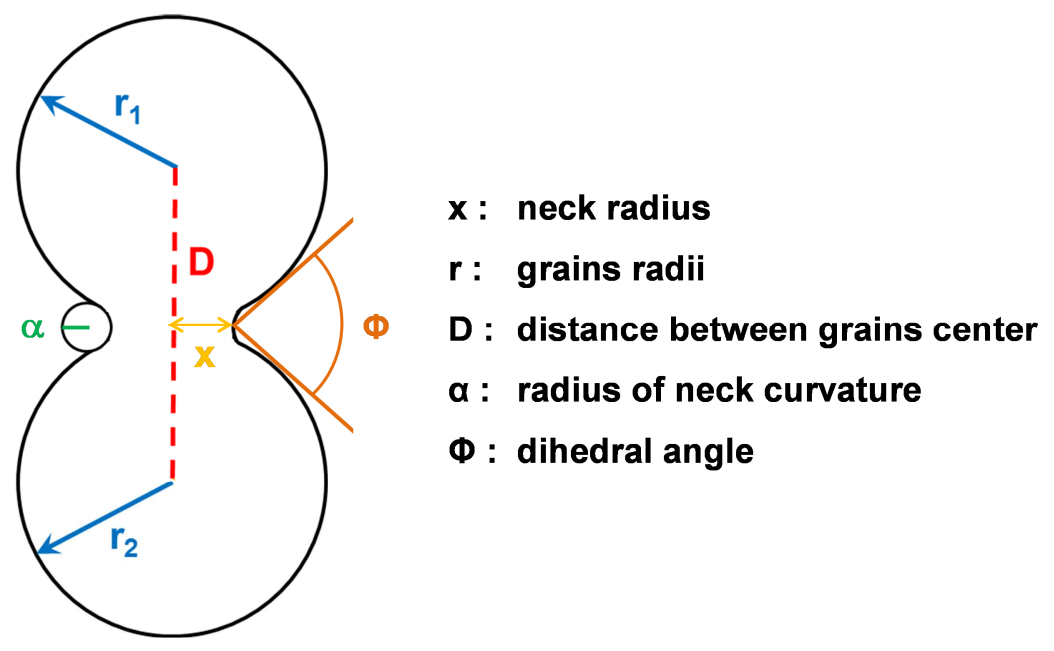

Figure 2 : Characteristic parameters retained to describe the first stage of sintering of a two-grain system.

\subsection{Numerical calculations}

The SALAMMBO code used for numerical calculations in this study is a model in development on a sub-granular scale (i.e. crystallographic grains of materials are meshed) for the simulation of solid state sintering of ceramics ${ }^{30}$. It aims at describing grain and pore size evolution during a free (or under gas pressure) sintering.

Free surfaces and grain boundary local changes in principal curvature radii induce an extra stress upon the outer boundaries of the grains. Grains are considered as single crystals with an elastic constitutive law. Navier-Lamé equations are solved in the bulk of each grain with, as von Neumann boundary conditions, the sum of the gas pressure and of the extra stress mentioned before. It gives the 3D-displacement field and hence the density of the elastic mechanical energy. 
Resulting changes in Gibbs free energy along the grain interfaces (free surfaces and grain boundaries) induce surface and grain boundary mass transport according to Fick's first law. Since matter is almost incompressible, locally accumulated matter on grain surfaces makes the surface move (second Fick's law). This results in an irreversible shape evolution of grains during the sintering fabrication stage.

\section{Results and discussion}

\subsection{Microstructural evolution}

The in situ HT-ESEM study of the first stage of $\mathrm{CeO}_{2}$ microspheres sintering allowed the direct observation of the morphological modifications occurring on a pair of grains during an isothermal heat treatment. Such modifications are clearly evidenced on the ESEM images series gathered in Figure $\mathbf{3}$ as an example for polycrystalline and single crystal particles $\left(\mathrm{T}=1050^{\circ} \mathrm{C}\right)$. Videos are also supplied as supplementary material for another couple of systems studied at $\mathrm{T}=1150^{\circ} \mathrm{C}$.

The microstructural modifications occurring on the assembly during sintering can be directly observed with a time resolution of 1 to 30 seconds. For both systems, a neck between the microspheres was first formed and then grew continuously. Same modifications occurred for the other sintering temperatures studied, i.e. in the $1100^{\circ} \mathrm{C}-1225^{\circ} \mathrm{C}$ range, but with different kinetics. Also, the neck growth was systematically more pronounced for the polycrystalline grains compared to single crystal systems in the same sintering conditions. For example, after 120 min. of heating at $1050^{\circ} \mathrm{C}$, the neck between polycrystalline grains of about $450 \mathrm{~nm}$ was about $250 \mathrm{~nm}$ in diameter while it only reached about $180 \mathrm{~nm}$ for single crystals of similar size (Figure 3). 


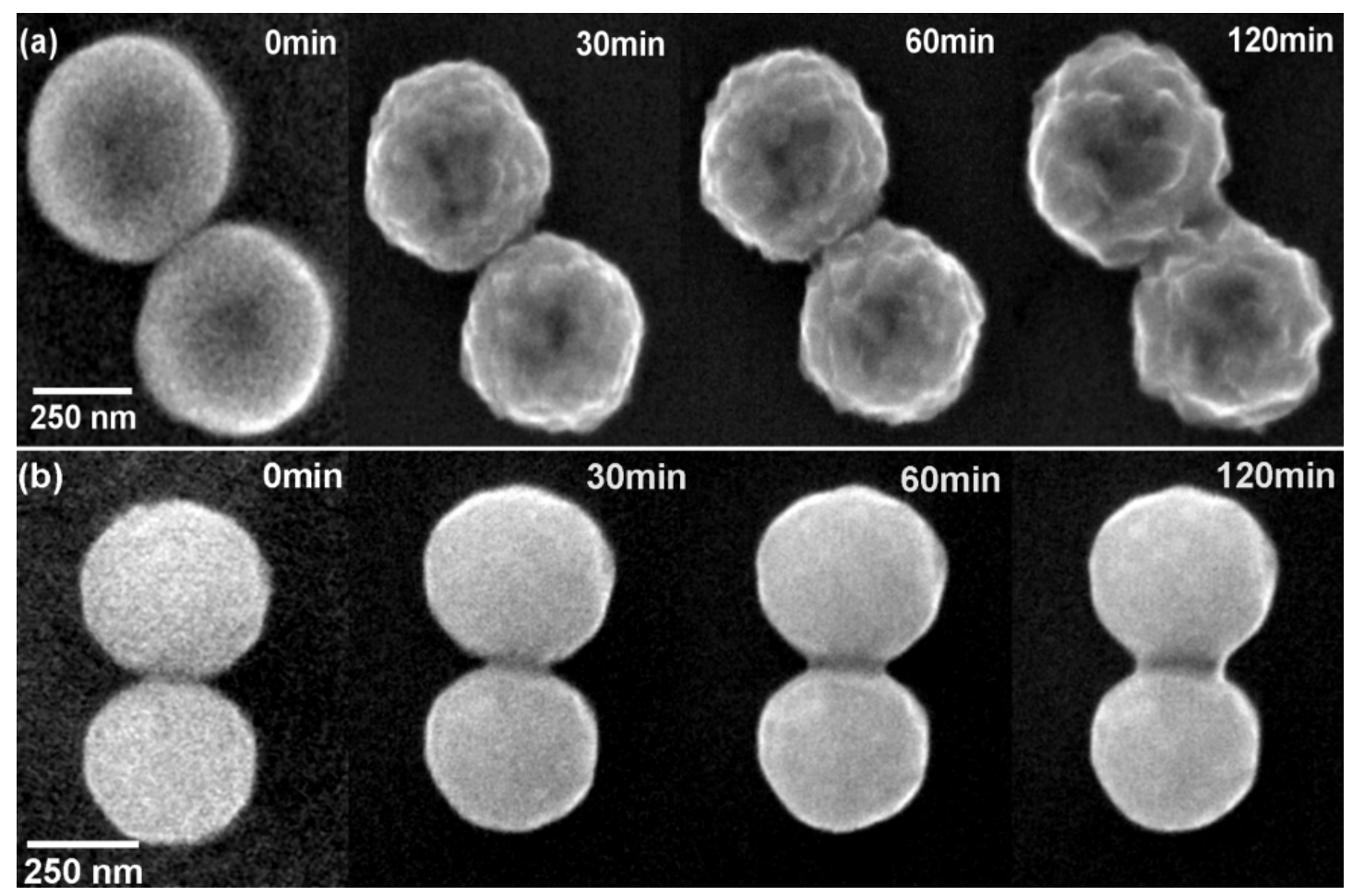

Figure 3 : In situ HT-ESEM observation of $\mathrm{CeO}_{2}$ microspheres sintering on a Pt-Au10 substrate at $1050^{\circ} \mathrm{C}$ : (a) polycrystalline material and (b) single-crystal particles.

Moreover, for the polycrystalline microspheres, neck growth was simultaneously accompanied by the gradual decrease of crystallites number within the grains. Indeed, after 30 min. of heat treatment at $1050^{\circ} \mathrm{C}$, the mean size of crystallites was about $25 \mathrm{~nm}$ whereas it could reach up to $100 \mathrm{~nm}$ after $120 \mathrm{~min}$. A similar behavior was already observed previously for the crystallites growth within isolated $\mathrm{CeO}_{2}$ microspheres ${ }^{22}$. For this system, a gradual decrease of the crystallites number was evidenced versus heating time, the mean size of crystallites shifting from about $50 \mathrm{~nm}$ after $30 \mathrm{~min}$ at $1050^{\circ} \mathrm{C}$ to $100 \mathrm{~nm}$ after $120 \mathrm{~min}$. Additionally, the observations performed in our study for a couple of particles revealed that when applying heat temperatures above $1150^{\circ} \mathrm{C}$, the crystallite number decreased rapidly until two single crystal grains in contact were obtained. 


\subsection{Evolution of parameters of interest}

All the ESEM images recorded for these systems at different temperatures were then processed to highlight the variation of the parameters of interest used for description of the first step of sintering.

As previously detailed, both Fiji and the homemade ImageJu software were used for image processing in order to determine for each image the values of several parameters of interest such as the neck size, grain radius, etc. (Figure 2). As instance, the evolution of these parameters recorded during a heat treatment at $1100^{\circ} \mathrm{C}$ is shown in Figure 4.

The evolution of neck radius during sintering is reported in Figure 4a for polycrystalline $(\mathrm{r} \approx$ $250 \mathrm{~nm})$ and single crystal particles $(\mathrm{r} \approx 200 \mathrm{~nm})$. In such conditions, it is useful to precise that the differences induced by the variation of particles diameter between the two systems studied remained negligible regarding to the uncertainty attached to the image analysis. For single crystals, the neck grows very fast during the first $10 \mathrm{~min}$. of heating then reaches a constant value of about $90 \mathrm{~nm}$. Conversely, at the same temperature, the neck that develops between polycrystalline grains grows continuously to reach $200 \mathrm{~nm}$ after $60 \mathrm{~min}$. of heating. The trend initially sketched from the HT-ESEM micrographs is thus confirmed and argues for a faster and more complete sintering of polycrystalline systems.

Simultaneously to neck growth, an increase of the angle formed by the tangents of the two grains in the neck region was also observed. For single crystal-based systems used in numerical models, this angle is generally referred to as the dihedral angle, and its value driven by the equilibrium of forces at the triple point between two crystals in contact and air. Hence, this value does not reflect a strict physical reality for polycrystalline systems since it exists as many dihedral angles as crystal/crystal/air junctions in the system. However, such dihedral angle like remains a very interesting value to consider when studying the sintering of two 
spherical particles with a geometrical approach. As stated for the neck's diameter, the increase in the angle values is also faster for polycrystalline grains than for single crystal microspheres (Figure 4b).
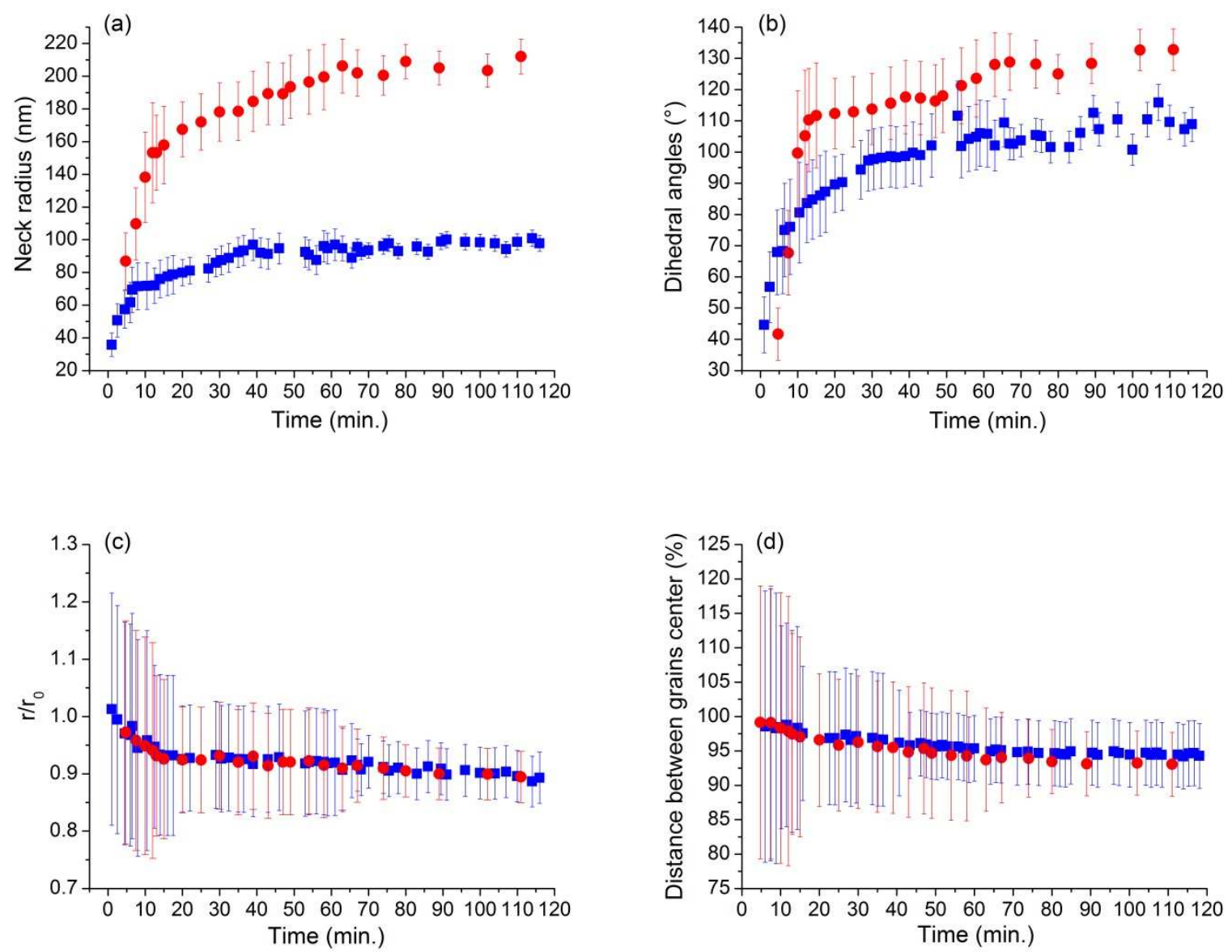

Figure 4 : Evolution of various parameters of interest during sintering of polycrystalline $(\bullet)$ and single crystal ( $\square$ ) $\mathrm{CeO}_{2}$ microspheres at $1100^{\circ} \mathrm{C}$ : (a) neck radius, (b) dihedral angles, (c) relative grains radii and (d) relative distance between the grain centers. On these curves, only one point out of ten is reported to provide a better readability of the results.

Conversely, the variation of the grains radii $\left(r_{1}\right.$ and $\left.r_{2}\right)$ and of the distance between the grains centers (D) is similar for both types of systems studied; these parameters are weakly, if not, dependent of the grains crystallinity. Indeed, the decrease in the relative grains radii $\left(r / r_{0}\right)$ is 
comparable for both systems and reaches about $10 \%$ after 100 min of sintering at $1100^{\circ} \mathrm{C}$ (Figure 4c). As detailed in a previous work ${ }^{22}$, such decrease in the grains size is mainly due to the inter-granular rearrangement as well as pores and defects elimination during heating for polycrystalline systems. In this case, pore and defects elimination is enhanced by grain boundary diffusion in the bulk of spheres.

In addition, the variation of the grains radii is accompanied by the slight decrease of distance between grains centers (Figure 4d) which accounts for the densification of the two grains system ${ }^{\mathbf{4}, 31}$. It is to be noted that such a phenomenon is generally associated with the second stage of sintering (characterized by the elimination of the open porosity), and responsible for the macroscopic shrinkage of the compacts. Nevertheless, the observations performed in situ during this study clearly evidence that the beginning of the densification is concomitant with the neck formation, i.e. that there is not a clear boundary between the first and second stages of sintering. As a matter of fact, a similar trend was also observed by Lange ${ }^{32}$ during the sintering of zirconium oxide compact with polycrystalline grains and single crystal grains assemblies.

\subsection{Neck growth mechanisms and associated activation energies}

During the first stage of sintering, the progression of the neck's elaboration is generally represented by a dimensionless parameter which makes it possible to compare the morphological changes occurring in systems constituted by grains with different sizes. The so-called sintering degree $(y)$ is then defined as the ratio of neck and grains radii $(y=x / r)^{3,33}$. In this study, the neck radius $(\mathrm{x})$ and the mean grains radius $\left(\mathrm{r}=\left(\mathrm{r}_{1}+\mathrm{r}_{2}\right) / 2\right)$ were determined by image analysis and the evolution of the sintering degree was plotted for both single crystal and polycrystalline microspheres systems (Figure 5). 
As expected, the evolution of the sintering degree appeared to be driven by the variation of neck size for both systems: whatever the experimental conditions explored, the sintering degree was thus systematically higher for the polycrystalline grains than for the single crystals. Such an observation appeared to be consistent with the early results reported by Slamovitch and Lange for $\mathrm{ZrO}_{2}$ spherical particles ${ }^{34}$. For polycrystalline particles, their microstructural observations showed that because the crystallites composing the microspheres exhibited growth, the crystallites embedded in the neck region were unable to maintain an equilibrium configuration. Hence, the polycrystalline system never reached a steady-state whereas single crystals rapidly formed a neck with constant size in the same sintering conditions. The crystallinity of the powders then turns out to be a very important parameter which influences the sintering behavior at the microscopic and macroscopic scales ${ }^{\mathbf{2 3 , 3 2}}$ and should be considered carefully.

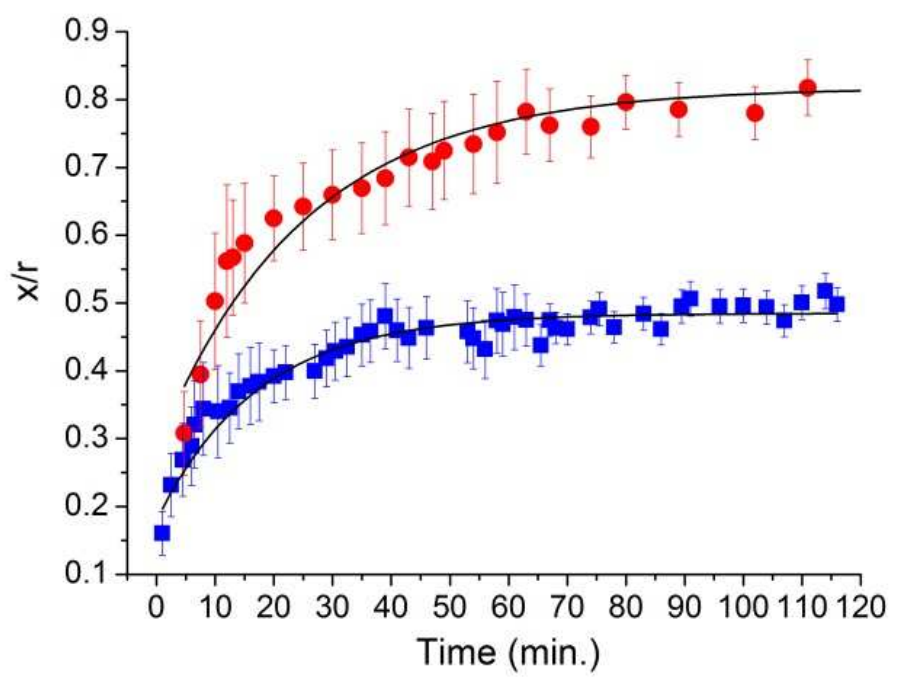

Figure 5 : Evolution of the sintering degree $(y=x / r)$ during sintering of two polycrystalline $(\bullet)$ and single crystal ( $\square) \mathrm{CeO}_{2}$ microspheres at $\mathrm{T}=1100^{\circ} \mathrm{C}$. Fitting of the data was performed using a simple exponential law for the polycrystalline grains and using the general kinetic law of neck growth for single crystal grains. 
In order to determine the sintering kinetics constants, the evolution of the sintering degree determined for each isothermal treatment $\left(1050^{\circ} \mathrm{C}-1225^{\circ} \mathrm{C}\right)$ was then fitted using different analytical sintering models. For single crystal particles, the general law of neck growth developed during the 1950's to model the first stage of sintering was used ${ }^{\mathbf{1 , 5 , 3}}$ :

$$
y^{n}=\frac{k \cdot t}{r^{m}}
$$

Where $\mathrm{y}=\mathrm{x} / \mathrm{r}$ denotes the sintering degree, $k$ is a kinetic constant, $\mathrm{t}$ is the duration of heat treatment, and $n$ and $m$ are exponents characteristic of the diffusion process involved (i.e. mainly surface, volume or grain boundary diffusion).

It is to be noted that such a model could also be used to describe the linear shrinkage of a pellet during sintering. Its application was massively used in the field of ceramic materials, since it allowed the identification of diffusion mechanisms and of kinetics parameters through macroscopic measurements (the Dorn's method, based on dilatometry, being probably one of the most common approach). In this study, the general law described above was used for the first time at the microscopic level and led to determine effective kinetics constants at different temperatures (Table 1).

The $\mathrm{k}$ values obtained were further plotted into an Arrhenius diagram in order to determine the activation energies corresponding to the first stage of sintering in the two systems considered. For the one based on single crystal particles, the activation energy was found to $516 \pm 27 \mathrm{~kJ} \mathrm{~mol}^{-1}$ (Figure 6a). This value is consistent with those previously reported in the literature for the sintering of bulk cerium dioxide. However, it is clearly located in the upper limit of the wide range of values reported by various authors and obtained under different experimental conditions ${ }^{\mathbf{2 5 , 3 5}}$. As a matter of example, Florio et al. determined a value of 325 $\mathrm{kJ} . \mathrm{mol}^{-136}$ whereas Chen et al. ${ }^{37}$ obtained a value about $580 \mathrm{~kJ} \cdot \mathrm{mol}^{-1}$. 
Table 1 : Kinetics constant obtained during the sintering of polycrystalline grains with exponential law $(y=$ $\left.a_{0} \cdot \exp (k t)+y_{0}\right)$ and single crystal grains with the law of neck growth $\left(y^{n}=\frac{k \cdot t}{r^{m}}\right)$.

\begin{tabular}{c|ccc|ccc}
\hline \multirow{2}{*}{$\begin{array}{c}\text { Temperature } \\
\left({ }^{\circ} \mathbf{C}\right)\end{array}$} & \multicolumn{3}{|c|}{ Single crystal grains } & \multicolumn{3}{c}{ Polycrystalline grains } \\
& $\mathrm{k}^{\prime}=\left(\mathrm{k} / \mathrm{r}^{\mathrm{m}}\right)^{1 / \mathrm{n}}$ & $\mathrm{k}$ & $\ln (\mathrm{k})$ & $\mathrm{a}_{0}$ & $\mathrm{k}$ & $\ln (\mathrm{k})$ \\
\hline 1050 & 0.17 & $2.26 \times 10^{4}$ & 10.03 & -62.87 & 0.016 & -4.14 \\
1100 & 0.22 & $1.75 \times 10^{5}$ & 12.07 & -28.01 & 0.035 & -3.33 \\
1150 & 0.29 & $6.24 \times 10^{5}$ & 13.34 & n.d. & n.d. & n.d. \\
1200 & 0.36 & $2.98 \times 10^{6}$ & 14.90 & -14.19 & 0.070 & -2.65 \\
1225 & n.d. & n.d. & n.d. & -6.67 & 0.15 & -1.89 \\
\hline
\end{tabular}

n.d. : not determined
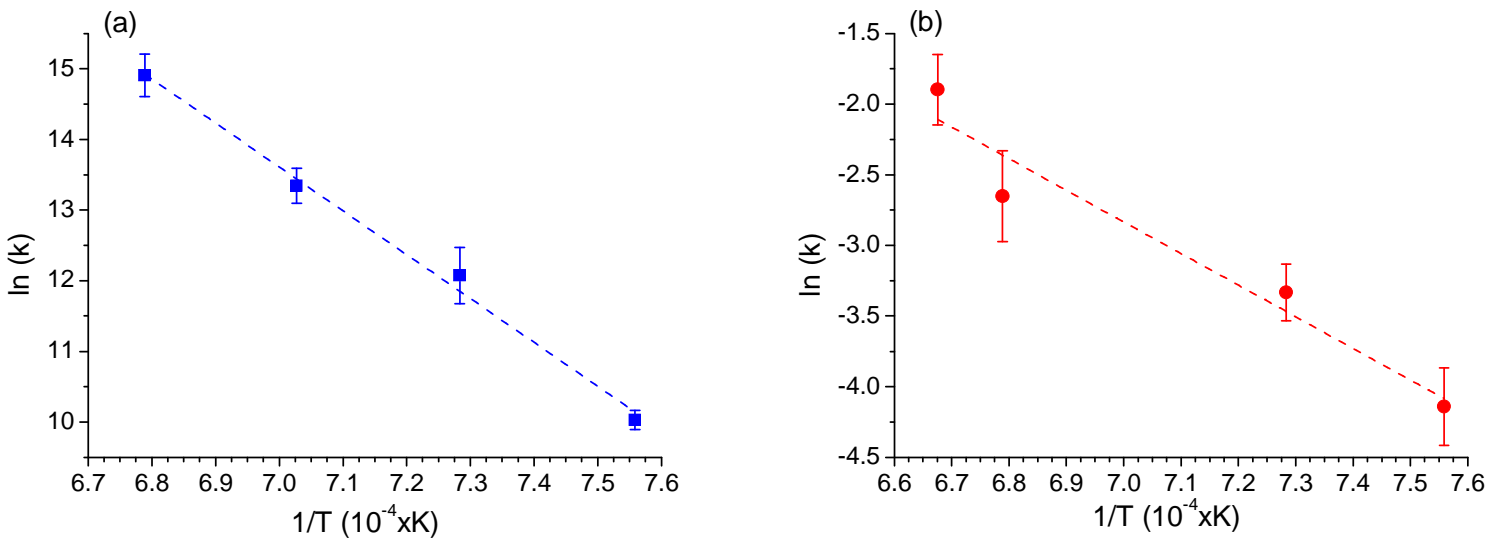

Figure 6 : Arrhenius diagram and activation energies determined during sintering at different temperature (between $1050^{\circ} \mathrm{C}-1225^{\circ} \mathrm{C}$ ) for (a) single crystal grains ( $(\bullet)$ and (b) polycrystalline grains $(\bullet)$.

For the polycrystalline grains-based system, the experimental data was modeled with an exponential function (Figure 5). Indeed, the general law previously used was developed based on the hypothesis of single crystals and cannot apply to polycrystals for which there is actually no model available in the literature. Hence, a simple first-order law was considered and allowed the determination of sintering kinetics: 


$$
y=a_{0} \cdot \exp (k t)+y_{0}
$$

Where $\mathrm{y}=\mathrm{x} / \mathrm{r}$ denotes the sintering degree, $\mathrm{y}_{0}$ the sintering degree à $\mathrm{t}=0, k$ the kinetic constant, $\mathrm{t}$ the duration of heat treatment and $\mathrm{a}_{0}$ a constant.

On this basis, the activation energy determined by using the Arrhenius diagram from the

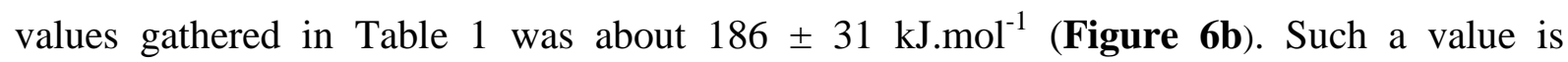
significantly lower than that determined for the single crystal grains system, indicating that the mechanisms involved in the neck's formation, and more generally in the global morphology's evolution, are different between the two systems considered.

For polycrystalline particles, activation energy is significantly lower than the values generally reported in the literature for the sintering of bulk $\mathrm{CeO}_{2}$, which are commonly associated to pure matter diffusion between the grains ${ }^{\mathbf{3 6 , 3 7}}$. Conversely, the value obtained in this work is more likely representative of two mechanisms occurring concomitantly (at least partially) during the heat treatment. At the beginning of heating, several crystallites with nanoscale size are present in the region of contact between the two grains. The formation of the neck is then probably driven by the reorganization of crystalline planes in the same orientation, which promotes the formation of a continuous lattice between the grains. This crystalline plane reorganization then leads to the formation of the neck between the grains constituted by many crystallites. Such a mechanical rearrangement was previously described by some authors and known as Oriented Attachment $(\mathrm{OA})^{38}$. For $\mathrm{CeO}_{2}$ the activation energy associated with this mechanism ranged between $52 \pm 4 \mathrm{~kJ} \cdot \mathrm{mol}^{-139}$ and $95 \pm 15 \mathrm{~kJ} \cdot \mathrm{mol}^{-122}$.

After its initial formation, the neck between the polycrystalline grains was still formed by several nanoscale crystallites. Indeed, the Oriented Attachment (OA) mechanism only led to a moderate growth of crystallites that retain a nanoscale size, and to a decrease of grains porosity. The crystallite mobility is thus less favorable and the growth of the neck is no longer 
driven by the Oriented Attachment (OA) but by diffusive processes between the two grains. As stated above, the activation energy reported in the literature for this second kind of mechanism is generally between $300 \mathrm{~kJ}^{\mathrm{mol}}{ }^{-1} \mathbf{4 0 , 4 1}$ and $600 \mathrm{~kJ} \cdot \mathrm{mol}^{-1}{ }^{36,37}$. As the global activation energy determined herein for the sintering of two polycrystalline particles (186 \pm $31 \mathrm{~kJ}^{\mathrm{mol}}{ }^{-1}$ ) is ranging between the values reported for Oriented Attachment and diffusion, both mechanisms probably occur simultaneously and continuously in our system. A similar combination of processes was also pointed out during the study of nano-ripening within $\mathrm{CeO}_{2}$ microspheres $^{22}$, which drives the growth of crystallites during heat treatments at high temperature. However, during this study, a combination of two exponentials had to be used to fit accurately the crystallites' growth kinetics, which accounted for the consecutive character of the different mechanisms operating. Conversely, the use of such two-exponentials model did not provide satisfying results when fitting the sintering degree evolution depicted in Figure 5. Hence, oriented attachment and diffusion are probably more intricate in this case : in some locations of the sample, oriented attachment could still occur while grain boundary diffusion is already operating elsewhere, which could be viewed as differences in the advancement state of the sintering process. Among other causes, this is likely to result from the decrease of liberty degree of crystallites induced by the neck formation.

For the single crystal particles, a significantly higher value of the activation energy was evidenced $\left(516 \pm 27 \mathrm{~kJ} \cdot \mathrm{mol}^{-1}\right)$. In this case, the Oriented Attachment $(\mathrm{OA})$ cannot occur notably because of the particles size (several hundredths of nanometers). So, the only way to process the neck growth is solid state diffusion. It thus corresponds to the well-known theoretical model where two spherical grains are in contact, and the formation and growth of the neck is ensured by mass transport from the grains to the neck area region. It was already noticed that our value is ranging in the upper limit of the data reported in the literature for $\mathrm{CeO}_{2}$ sintering (typically from 300 to $600 \mathrm{~kJ} \mathrm{~mol}^{-125,35}$ ). As such a wide range of values 
cannot be explained only by considering a variable set of operating conditions (particularly in terms of heat temperature and atmosphere), it is thus very likely that the polycristallinity of the powdered samples used during these experiments, along with the potential inner porosity of the microspheres, impacts strongly the activation energy of sintering.

\subsection{Material transport path}

Five different diffusion processes are usually supposed to drive sintering: surface diffusion, volume diffusion, grain boundary diffusion, evaporation-condensation and viscous flow ${ }^{\mathbf{4 2 , 4 3}}$. Among these mass transport mechanisms, only three lead to the densification of the sample (i.e. for two grains systems, the decrease of distance between the centers of the grains): grain boundary diffusion, volume diffusion and viscous flow for the liquid phase sintering. The identification of the diffusion process during cerium oxide sintering can be obtained by using the general kinetics law of neck growth and subsequent determination of the $n$ value (3). Indeed, the $\mathrm{n}$ value is specific for a given diffusion mechanism with: $n=2$ for viscous flow; $n$ $=3$ for evaporation-condensation; $n=4$ for volume diffusion; $n=7$ for surface diffusion and

$n=6$ for grain boundary diffusion ${ }^{44,45}$. In our case, the $n$ value can be determined according to equation (3).

$$
y=\left(\frac{k \cdot t}{r^{m}}\right)^{1 / n}=\left(k^{\prime} \cdot t\right)^{1 / n}
$$

In this latter, the $\mathrm{r}^{\mathrm{m}}$ parameter is supposed to remain almost unchanged, in good agreement with the weak variation stated from image analysis in all our operating conditions, which led $\mathrm{k}^{\prime}=\mathrm{k} / \mathrm{r}^{\mathrm{m}}$ to be constant. In the case of the system constituted by single crystalline grains, the values of the $n$ exponent determined at different sintering temperatures (Table 2) appeared to 
be systematically close to 6 . As a matter of fact, the sintering of two single crystal $\mathrm{CeO}_{2}$ microspheres is then driven by a grain boundary diffusion mechanism (Figure 4).

Table 2 : Values of $\boldsymbol{n}$ exponent determined during sintering of single crystal grains by using the general kinetic law of neck growth $\left(y^{n}=\frac{k \cdot t}{r^{m}}\right)$.

\begin{tabular}{cc}
\hline Temperature $\left({ }^{\circ} \mathrm{C}\right)$ & $N$ \\
& \\
\hline 1050 & $5.3 \pm 0.9$ \\
1100 & $5.8 \pm 0.7$ \\
1150 & $6.5 \pm 0.8$ \\
1200 & $5.5 \pm 0.6$ \\
\hline
\end{tabular}

The general kinetic law of neck growth previously used for the identification of diffusion mechanism during sintering of single crystal grains systems cannot be applied in the case of sintering of polycrystalline particles. Thus, for such systems, the Herring's scaling law was used for the identification of the main mass transport mechanism operating during sintering. Indeed, this law (4) considers the particles size influence on the microstructural modifications of the sample during sintering. For the powders (or the grains) with similar shapes and different sizes under the same sintering experimental conditions, the Herring's scaling law predicts the relative time required to reach the same sintering degree ${ }^{46}$. During sintering of two systems constituted by two spherical grains in contact with different radii, the ratio between the times required to reach the same degree of sintering can be expressed as ${ }^{\mathbf{4 7 , 4 8}}$ :

$$
\frac{t_{1}}{t_{2}}=\left(\frac{r_{1}}{r_{2}}\right)^{m}
$$


Where $r_{1}$ and $r_{2}$ are the grains radii, $t_{1}$ and $t_{2}$ the sintering times, and $m$ an exponent specific to the diffusion mechanism operating during sintering, with $m=1$ for viscous flow; $m=2$ for evaporation-condensation; $m=3$ for volume diffusion and $m=4$ for surface diffusion or grain boundary diffusion.

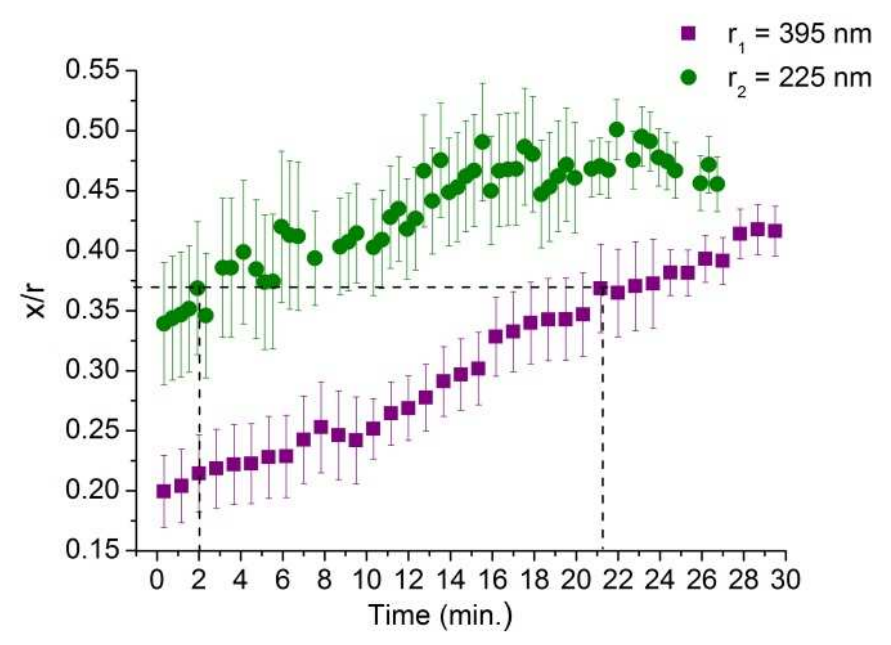

Figure 7 : Evolution of sintering degree $(\mathrm{x} / \mathrm{r})$ during heating $\left(\mathrm{T}=1200^{\circ} \mathrm{C}\right)$ of two polycrystalline $\mathrm{CeO}_{2}$ microspheres with different sizes: $(\square) r_{1}=395 \pm 10 \mathrm{~nm}$ and $(\bullet) r_{2}=225 \pm 10 \mathrm{~nm}$.

Therefore, the Herring scaling law was applied on two polycrystalline grains systems constituted by grains with different size $\left(r_{1}=395 \pm 10 \mathrm{~nm}\right.$ and $\left.r_{2}=225 \pm 10 \mathrm{~nm}\right)$ and sintered at $1200^{\circ} \mathrm{C}$ (Figure 7). For different given sintering degrees (i.e. constant $\mathrm{y}=\mathrm{x} / \mathrm{r}$ value), the value of the $m$ exponent was calculated and a value close to 4 was systematically obtained (Table 3). As for single crystals, the prevailing sintering mechanism is thus grain boundary diffusion for polycrystalline grains. In these conditions, the polycristallinity of the particles appeared to only influence the main type of processes operating during neck elaboration. Indeed, sintering nanocrystalline aggregates will result in a strong contribution of oriented attachment that tends to lower the global activation energy. However, it is not expected to modify the preferential diffusion path used to process mass transport. 
Table 3 : Values of $m$ exponent determined during the sintering of polycrystalline grains by using the Herring scaling law $\left(\frac{t_{1}}{t_{2}}=\left(\frac{r_{1}}{r_{2}}\right)^{m}\right)$.

\begin{tabular}{cccc}
\hline Sintering degree $(\mathrm{x} / \mathrm{r})$ & $\mathrm{t}_{1}(\mathrm{~min})$ & $\mathrm{t}_{2}(\min )$ & $m$ \\
\hline 0.35 & 1.6 & 20.4 & $4.3 \pm 0.7$ \\
0.37 & 2 & 21.3 & $4 \pm 0.5$ \\
0.39 & 3 & 26.5 & $3.8 \pm 0.5$ \\
\hline
\end{tabular}

\subsection{Modelling of $\mathrm{CeO}_{2}$ sintering}

As already exposed, the main differences in the microstructural modifications occurring in the polycrystalline and single crystal particles systems is reflected by the significant variation of sintering degree between both systems for a given time. For all the operating conditions tested, the evolution of neck size almost reached steady-state for single crystals while the neck formed between polycrystalline particles kept growing all along the heat treatment. Such a difference leads to a sintering degree that was generally twice higher for polycrystalline systems compared to the reference single crystals. On this basis, a comparison with the evolution of sintering degree calculated by numerical modeling was also performed during this work in order to evaluate the accuracy of such simulations in the prediction of the kinetics linked with the first stage of sintering. In this aim, a predictive model using the SALAMMBO code ${ }^{\mathbf{4 9 , 5 0}}$ was used. This latter was implemented with the data gathered in the Table $4^{\mathbf{5 1}}$. Along with the experimental conditions used (temperature) and the topological parameters evaluated through image analysis to represent the initial geometry of grains in contact, physical quantities coming from the literature were used. Herein, $\delta \mathrm{D}_{\mathrm{b}}$ stands for the product of the grain boundary diffusion coefficient with the grain boundary thickness, and was calculated from the value of grain boundary grooving kinetics reported by Chen for $\mathrm{CeO}_{2}$ at $1270^{\circ} \mathrm{C}^{\mathbf{5 2}}$. 
Similarly, $\delta \mathrm{D}_{\mathrm{S}}$ corresponds to surface diffusion and was estimated from the relation established by Jin et al. in the $1200-1390^{\circ} \mathrm{C}_{\text {range }}{ }^{\mathbf{5 3}}$ :

$$
D_{S}=(35 \pm 1.6) e^{-\frac{E_{A}}{R T}} \quad\left(\text { in } m^{2} . s^{-1}\right)
$$

with $\mathrm{E}_{\mathrm{A}}=458 \pm 14 \mathrm{~kJ} \cdot \mathrm{mol}^{-1}$. The molecular volume of the diffusion species, $\Omega$, and the surface tension, $\gamma_{\mathrm{S}}$, were taken from the same work. In this latter case, as $\gamma_{\mathrm{S}}$ varies from 1.12 to $2.43 \mathrm{~J}_{\mathrm{m}} \mathrm{m}^{-2}$ with the crystalline orientation, an upper value was considered.

Table 4 : Input data used for modelling of single crystal grains sintering.

\begin{tabular}{|c|c|c|c|c|}
\hline 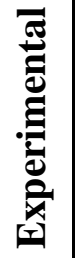 & $\begin{array}{c}\text { Temperature }\left({ }^{\circ} \mathrm{C}\right) \\
1100\end{array}$ & Grain radii (nm) & $\begin{array}{c}\text { Initial neck size }(\mathrm{nm}) \\
47\end{array}$ & \\
\hline : & $\begin{array}{c}\delta \mathrm{D}_{\mathrm{b}}\left(\mathrm{m}^{3} \cdot \mathrm{s}^{-1}\right)^{52} \\
10^{-24}\end{array}$ & $\begin{array}{c}\delta \mathrm{D}_{\mathrm{S}}\left(\mathrm{m}^{3} \cdot \mathrm{s}^{-1}\right)^{\mathbf{5 3}} \\
10^{-24}\end{array}$ & $\begin{array}{c}\Omega\left(\mathrm{m}^{3}\right)^{\mathbf{5 2}} \\
10^{-29}\end{array}$ & $\begin{array}{c}\gamma_{\mathrm{S}}\left(\mathrm{J} \cdot \mathrm{m}^{-2}\right)^{54} \\
2.43\end{array}$ \\
\hline
\end{tabular}

The comparison between modelling and experimental results for the single crystal particles evidenced a very good correlation between the two sets of data (Figure 8). Indeed, the evolution of sintering degree followed a similar trend despite slight differences in the absolute values of $y$. Such small discrepancies could mainly be assigned to the morphology of the particles used in the experimental study which were evidently not perfectly spherical. Apart from this negligible bias, the code used for the calculations thus appeared to fit very nicely the experimental results. On this basis, it could be used to forecast the behavior of $\mathrm{CeO}_{2}$ single crystal microspheres in other operating conditions, mainly in terms of heating time and temperature. Also, its application could probably be extended to other materials; including 
isostructural actinides-based oxides ( $\mathrm{such}$ as $\mathrm{ThO}_{2}, \mathrm{UO}_{2}$ and $\mathrm{PuO}_{2}$ ) which are of great interest in the nuclear fuel cycle although they remain difficult to handle experimentally.

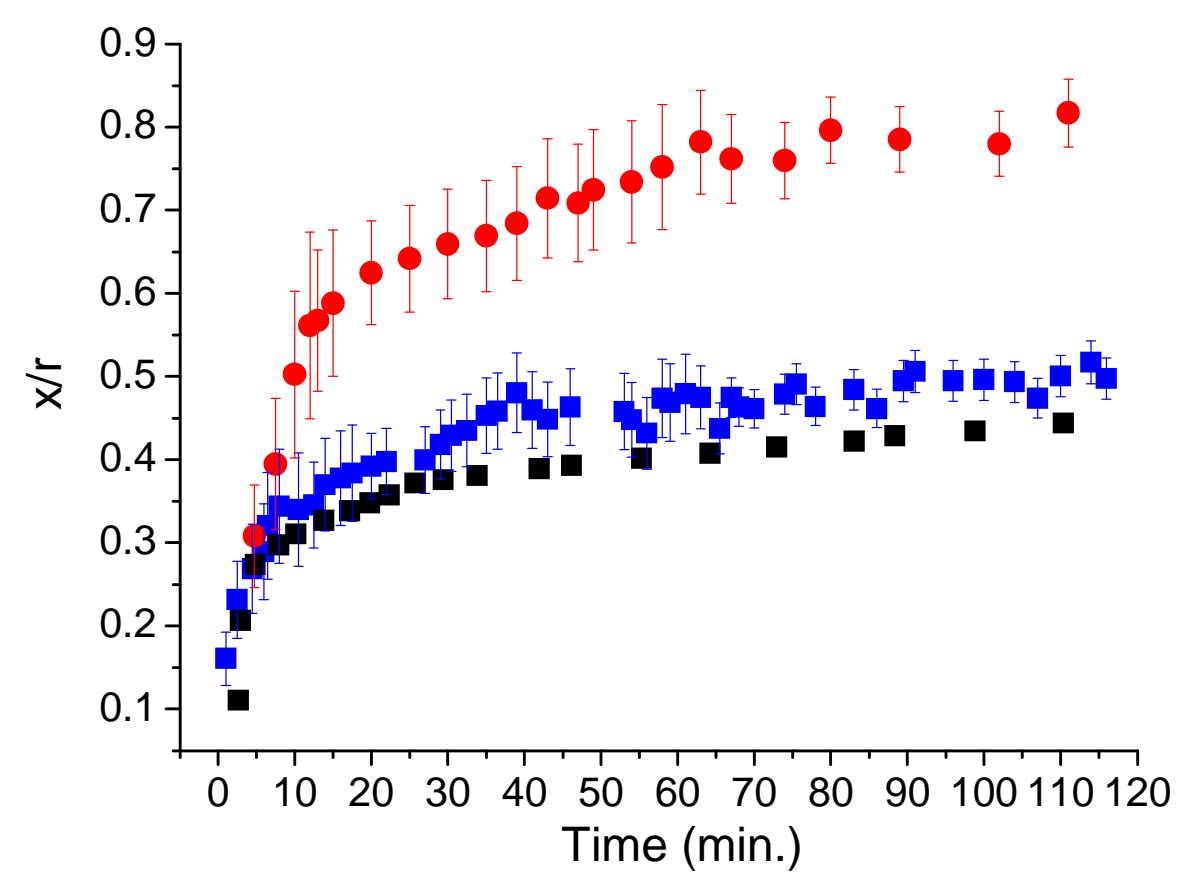

Figure 8 : Comparison between experimental results and modelling during sintering of two $\mathrm{CeO}_{2}$ microspheres at $\mathrm{T}=1100^{\circ} \mathrm{C}:(\bullet)$ polycristalline grains, ( $\left.\square\right)$ single crystal grains and $(\square)$ modelling of single crystal grains sintering.

Conversely, and as expected from the results previously reported above, the simulated curve exhibited a significant deviation from the polycrystalline particles, i.e. from what is probably closest to a real material, even if the trends depicted for both data sets remain similar. This difference once again confirms the influence of the polycrystallinity of the grains on the sintering evolution, due to the contribution of mechanical reorganization of the crystallites in the neck region and to grain boundaries internal to spherical particles which act as diffusion short-circuits. On this basis, the model developed could for now only provide qualitative guidelines, but will not result in a reliable and quantitative prediction of the sintering degree evolution and morphology's variation. 
Both rearrangement and simulation of a great number of particles would be necessary for such a simulation. Such a work has already been undertaken by Martin with a simplified crystallite description Erreur! Signet non défini. Another model has been designed and developed by PinoMunoz et al. ${ }^{\mathbf{5 5}}$ in order to simulate the evolution of crystallites in contact taking into account diffusions mechanisms based on the level-set method. It already handles correctly a big number of crystallites (typically up to 100) but without a full coupling between grain boundary, surface and bulk diffusion. It recently ${ }^{\mathbf{5 6}}$ has taken into account chemical interdiffusion but, in the same way as SALAMMBO, this model does not account for crystallites initial re-arrangement (oriented attachment). 


\section{Conclusion}

The use of in situ HT-ESEM as a tool for the investigation of the first stage of sintering of $\mathrm{CeO}_{2}$ microspheres allowed the first direct observation of the morphological modifications of the samples during isothermal heat treatment. During these in situ observations a difference of evolution between the polycrystalline grains and single crystal grains was clearly observed. Moreover, advanced image processing allowed the determination of original and fundamental kinetics data that are typical of the processes occurring during the initial stage of densification in both cases: $186 \pm 31 \mathrm{~kJ}^{\mathrm{mol}}{ }^{-1}$ for polycrystalline grains and $516 \pm 27 \mathrm{~kJ}^{\mathrm{mol}}{ }^{-1}$ for single crystal grains.

The interpretation of the activation energies values led to the identification of the sintering mechanism: the Orientated Attachment (OA) and the diffusion for polycrystalline grains and pure diffusion for the single crystal grains. That is to say that the presence of several crystallites in the grains will induce a rapid evolution of the sintering degree due to the fast formation of contacts between crystallites in the neck region.

In addition, the comparison of experimental data and modelling shows a good correlation between the experimental data and modeling for the single crystal grains. But also the difference between sintering degree of polycrystalline grains and single crystal grains obtained by modeling. So, the single crystal grains generally used as sintering simplified model is not representative of the evolution of the polycrystalline grains systems, generally involved during sintering of ceramic materials. It is therefore important to take into account the presence of several crystallites during modelling of the first stage of sintering, for a better description of material aggregates behavior. 
Acknowledgements. Authors would like to thank the Materials Federative Project included in the NEEDS program (Nucléaire, Energie, Environnement, Déchets, Société) of CNRS for its continuous financial support.

Supporting Information Available. Videos obtained from the HT-ESEM observation of single-crystal and polycrystalline microspheres sintering at $\mathrm{T}=1150^{\circ} \mathrm{C}$. This information is available free of charge via the Internet at http://pubs.acs.org 
Table of Content Graphic

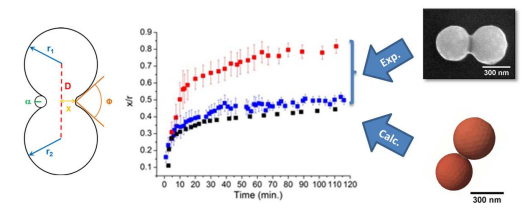




\section{References}

1. German, R.M. Sintering: from Empirical Observations to Scientific Principles, Elsevier ButterworthHeinemann, Oxford, U.K., 2014.

2. Kuczynski, G.C. Physics and chemistry of sintering, Adv. Coll. Interface Sci., 1972, 3, 275-330.

3. Kang, S.J.L. Sintering: Densification Grain Growth and Microstructure, Elsevier Butterworth-Heinemann, Oxford, U.K., 2005.

4. Kuczynski, G.C. The Mechanism of Densification During Sintering of Metallic Particles, Acta Metall., 1956, 4, 58-61.

5. Bernache-Assolant, D. Chimie-physique du frittage, Hermes, Paris, France, 1993.

6. Kuroiwa, D. A Study of Ice Sintering, Tellus, 1961, 13, 252-259.

7. Thomas, G.: Fulrath, R.M. Electron microscopy and structure of materials : proceedings, University of California Press, Berkeley, U.S.A., 1971.

8. Wakai, F.; Brakke, K.A. Mechanics of Sintering for Coupled Grain Boundary and Surface Diffusion, Acta. Mater., 2011, 59, 5379-5387.

9. Raut, J.S.; Bhagat, R.B.; Fichthorn, K.A. Sintering of Alumina Nanoparticles: A Molecular Dynamics Study, Nanostruct. Mater., 1998, 10, 837-851.

10. Zhang, W.; Schneibel, J.H. Sintering of Two Particles by Surface and Grain Boundary Diffusion - A Two Dimensional Numerical Study, Acta Metall. Mater., 1995, 43, 4377-4386.

11. Wakai, F. Modeling and Simulation of Elementary Process in Ideal Sintering, J. Am. Ceram. Soc., 2006, 89, 1471-1484.

12. Bordère, J.S.; Bernard, D. Full Resolution of the Monte Carlo Iime Scale Demonstrated through the Modelling of TwoAmorphousParticles Sintering, Comput. Mater. Sci., 2008, 43, 1074-1080.

13. Martin, S.; Parekh, R.; Guessasma, M.; Lechelle, J.; Fortin, J.; Saleh, K. Study of Sintering Kinetics of Bimodal Powders : a Parametric DEM Study, Powder Technol., 2015, 270, 637-645.

14. Pan, H.; Ko, S.H. The Solid State Neck Growth Mechanisms in Low Energy Laser Sintering of Gold Nanoparticles: a Molecular Dynamics Simulation Study, J. Heat Transf., 2008, 130, 1-7. 
15. Podor, R.; Ravaux, J.; Brau, H.P. in Scanning Electron Microscopy, Viacheslav Kazmiruk Ed, InTech, Rijeka, Croatia, 2012, 3, 31-54.

16. Knowles, R.W.; Hardt, T.A. High Temperature Specimen Stage and Detector for an Environmental Scanning Electron Microscope, EU Pat. EP 1003200 B1, 1996.

17. Gregori, J.; Kleebe, H.J.; Siegelin, F.; Ziegler, G. In Situ SEM Imaging at Temperatures as High as $1450^{\circ} \mathrm{C}$, J. Elec. Microsc., 2002, 54, 347-352.

18. Jacobs, G.; Williams, L.; Graham, U.; Sparks, D.; Davis, B.H. Low-Temperature Water-Gas Shift: In-Situ DRIFTS-Reaction Study of a $\mathrm{Pt} / \mathrm{CeO}_{2}$ Catalyst for Fuel Cell Reformer Applications, J. Phys. Chem. B, 2003, 107, 10398-10404.

19. Hirano, M.; Kato, E. Hydrothermal Synthesis of Nanocrystalline Cerium(IV) Oxide Powders, J. Am. Ceram. Soc., 2011, 50, 7150-7161.

20. Kim, H.S.; Joung, C.Y.; Lee, B.H.; Oh, J.Y.; Koo, Y.H.; Heimgartner, P. Applicability of $\mathrm{CeO}_{2}$ as a Surrogate for $\mathrm{PuO}_{2}$ in a MOX Fuel Development, J. Nucl. Mater., 2008, 378, 98-104.

21. Minamidate, Y.; Yin, S.; Sato, T. Synthesis of Monodispersed Rod-like and Spherical $\mathrm{CeO}_{2}$ Particles by Mild Solution Process, Mater. Sci. Eng., 2009, 1, 012003.

22. Nkou Bouala, G.I.; Clavier, N.; Léchelle, J.; Mesbah, A.; Dacheux, N.; Podor, R. In Situ HT-ESEM Study of $\mathrm{CeO}_{2}$ Nano-Ripening : Toward a Control of Nanostructure, Ceram. Intern., 2015, 41, 14703-14711.

23. Anumol, E.A.; Viswanath, B.; Ganesan, P.G.; Shi, Y.; Ramanath, G.; Ravishankar, N. Surface Diffusion Driven Nanoshell Formation by Controlled Sintering Mesoporous Nanoparticle Aggregates, Nanoscale, 2012, $2,1423-1425$

24. Joly-Pottuz, L.; Bogner, A.; Lasalle, A.; Malchere, A.; Thollet, G.; Deville, S. Improvements for Imaging Ceramics Sintering in situ in ESEM, J. Microsc., 2011, 244, 93-100.

25. Podor, R.; Clavier, N.; Ravaux, J.; Claparède, L.; Dacheux, N.; Bernache-Assollant, D. Dynamic Aspects of Cerium Dioxide Sintering: HT-ESEM Study of Grain Growth and Pore Elimination, J. Eur. Ceram. Soc., 2012, 32, 353-362.

26. ImageJ: Wayne Rasband, http://rsbweb.nih.gov/ij/ (accessed 12.16.2015); Fiji: Johannes Schindelin and al. http://fiji.sc/Fiji (accessed 12.16.2015). 
27. Abramoff, M.D.; Magalhães, P.J.; Sunanda, R.J. Image Processing with ImageJ, Biophotonics Intern., 2004, 11, 36-42.

28. Pascau, J.; Mateos Pérez, J.M. Image Processing with ImageJ, Packt publishing Ltd, Birmingham, U.K., 2013.

29. Favrichon, J. / Imageju, https://github.com/jfavrichon/imageju (accessed 12.16.2015).

30. Léchelle, J.; Martin, S.; Boyer, R.; Saikouk, K. A Sub-granular Scale Model for Solid-state Free Sintering : Results on the Evolution of Two Grains, J. Chem. Technol. Metall., 2014, 49, 263-274.

31. Swinkels, F.B.; Ashby, M.F. A Second Report on Sintering Diagrams, Acta Metall., 1981, 29, $259-281$.

32. Lange, F.F. Densification of Powder Compacts: An Unfinished Story, J. Eur. Ceram. Soc., 2008, 28, 15091516.

33. Lee, W.K.; Eadie, R.L.; Weatherly, G.C.; Aust, K.T. A Study of the Sintering of Spherical Silver PowderII. The Initial Stage, Acta Metall., 1978, 26, 1837-1843.

34. Slamovich, E.B.; Lange, F.F. Densification Behavior of Single Crystal and Polycrystalline Spherical Particles Zirconia, J. Am. Ceram. Soc., 1990, 73, 3368-3375.

35. Clavier, N.; Podor, R.; Deliere, L.; Ravaux, J.; Dacheux, N. Combining in situ HT-ESEM Observations and Dilatometry: An Original and Fast Way to the Sintering Map of $\mathrm{ThO}_{2}$, Mater. Chem. Phys., 2013, 137, 742749.

36. Zhang, T.; Hing, P.; Huang, H.; Kilner, J. Sintering and Grain Growth of CoO-doped $\mathrm{CeO}_{2} \mathrm{Ceramics} J$. Europ. Ceram. Soc., 2002, 22, 27-34.

37. Chen, P.L.; Chen, I. Grain Growth in $\mathrm{CeO}_{2}$ : Dopant Effects, Defect Mechanism, and Solute Drag, J. Am. Ceram. Soc., 1996, 79, 1793-1800.

38. Zhang, J.; Huang, F.; Lin, Z. Progress of Nanocrystalline Growth Kinetics Based on Oriented Attachment, Nanoscale, 2009, 2, 18-34.

39. Scardi, P.; Leoni, M.; Muller, M.; Maggio, R.D. In situ Size-strain Analysis of Nanocrystalline Ceria Growth Mater. Sci. Eng., 2010, 528, 77-82.

40. de Florio, D.Z.; Esposito, V.; Traversa, E.; Muccillo, R.; Fonseca, F.C. Master Sintering Curve for Gddoped $\mathrm{CeO}_{2}$ Solid Electrolytes, J. Therm. Anal. Calorim., 2009, 97, 143-147. 
41. Zhang, T.; Hing, P.; Huang, H.; Kilner, J. Early-stage Sintering Mechanisms of Fe-doped CeO ${ }_{2}$, J. Mater. Sci., 2002, 37, 997-1003.

42. Handwerker, C.A. PhD thesis, Massachusetts Institute of Technology, U.S.A. 1983.

43. Gessinger, G.H. Volume Diffusion as Densification Rate-controlling Step in Sintering, Scripta Metall., 1970, 4, 673-675.

44. Coble, R.L. Initial Sintering of Alumina and Hematite, J. Am. Ceram. Soc., 1958, 41, 55-61.

45. Ashby, M.F. A First Report on Sintering Diagrams, Acta Metall., 1974, 22, 275-289.

46. Herring, C. Effect of Change of Scale on Sintering Phenomena, J. Appl. Phys., 1950, 21, 301-303.

47. Kothari, N.C. The Effect of Particle Size on Sintering Kinetics in Alumina Powder, J. Nucl. Mater., 1965, $17,43-53$.

48. Lequitte, M.; Autissier, D. Synthesis and Sintering of Nanocrystalline Erbium Oxide, Nanostruct. Mater., 1995, 6, 333-336.

49. Ajdour, M. PhD thesis, Ecole des Mines de St-Etienne, France, 2006.

50. Ajdour, M.; Léchelle, J.; Valdivieso, F.; Goeuriot, P.; Saikouk, K.; Boyer, R. Sintering Simulation at a Scale Lower than the Grain Size, Adv. Sci. Technol., 2006, 45, 516-521.

51. Martin, S. PhD Thesis, Université de Technologie de Compiègne, France, 2014.

52. Chen, I.W. Grain Boundary Kinetics in Oxide Ceramics with the Cubic Fluorite Crystal Structure and its Derivative, Interf. Sci., 2000, 8, 147-156.

53. Jin, M.; Shimada, E.; Ikuma, Y. Atomic Force Microscopy Study of Surface Diffusion in Polycristalline $\mathrm{CeO}_{2}$ via Grain Boundary Grooving, J. Ceram. Soc. Jap., 2000, 5, 456-461.

54. Feral-Martin, C. PhD Thesis, Université Bordeaux 1, France, 2010.

55. Pino-Munoz, D.; Bruchon, J.; Drapier, S.; Valdivieso, F. Sintering at Particle Scale: An Eulerian Computing Framework to Deal with Strong Topological and Material Discontinuities, Arch. Comput. Method. E, 2014, 21, 141-187.

56. Tossoukpe, H. PhD Thesis, Ecole des Mines de St-Etienne, France, 2013. 